\title{
Lateral spread of MEMS WDM technologies
}

Hiroshi Toshiyoshi

Hiroshi Toshiyoshi, "Lateral spread of MEMS WDM technologies," Proc. SPIE 7930, MOEMS and Miniaturized Systems X, 793003 (11 February 2011); doi: $10.1117 / 12.882725$

SPIE. Event: SPIE MOEMS-MEMS, 2011, San Francisco, California, United States 


\title{
Lateral Spread of MEMS WDM Technologies
}

\author{
Hiroshi Toshiyoshi \\ Research Center for Advanced Science and Technology, The University of Tokyo, 4-6-1 Komaba, \\ Meguro-ku, Tokyo 153-8904, Japan
}

\begin{abstract}
Optical MEMS technologies originally developed for the WDM systems have found a wide range of lateral spreading applications. For instance, we have constructed a novel power-over-fiber type OCT endoscope by using two different wavelengths for powering an electrostatic MEMS scanner and for optical probing; this work is on the extension of a MEMS variable optical attenuator. Another example is a Fabry-Perot interferometer for wavelength filtering that has been redirected to a new use of a tunable color pixel developed in a plastic sheet of large area. We look into the diverging potential of MEMS in micro optics.
\end{abstract}

Keywords: MEMS, fiber optic application, VOA, projection display, endoscope

\section{INTRODUCTION}

History of optical MEMS (micro electro mechanical systems) goes back to the micro mirror devices for a projection display application reported by Westinghouse Inc. in 1975 [1]. At that time, no CMOS-MEMS technology was available and hence they used cathode ray in the vacuum tube to individually address and actuate micro mirrors. In 1980, Texas Instruments developed a deformable micro mirror device with electrical addressing circuit [2], which lead to the digital light processing devices today [3]. Ever since these inventions, compatibility of MEMS and micro optics has been acknowledged, and various applications have been investigated. Figure 1 shows the principles of light modulation where micro mechanisms are involved. Reflection on a micro mirror or refraction through a micro lens is the most classic spatial light modulation (SLM) method, and has found many industrial applications including fiber optic switch and image projection display. In the wave optics domain, we also can find diverging styles of opto-mechanical modulations such as wave-front control with a deformable mirror, diffraction control with mechanically movable gratings, wavelength tuning through a tilting etalon or a gap-controlled Fabry-Perot interferometer. Even sub-micron scale mechanical motion has a significant effect in optics due to the wave nature of light. Mechanical light processing further goes up to the electromagnetic domain, where polarization of light is tuned by nanowire grids or evanescent field of light is modulated by an optical absorber brought into a close vicinity of the light traveling in the waveguide. In recent years, electrons and photons are controlled by means of mechanical motion to bring the wavelength tuning capabilities into semiconductor laser diodes and photo detectors. Mechanical motions are usually slower than those of solid-state optical modulators; nonetheless, MEMS versions of optical modulation are used for those applications that need large deflection angles and complete block-out of light, for instance, which could not be done by the conventional solid-state solutions.

The author's research group at the University of Tokyo has spent more than 10 years to develop applications of optical MEMS technology in close collaboration with industrial partners. The first commercially released product was a variable optical attenuators (VOA) using an electrostatic micro mirror for wavelength division multiplex (WDM) systems. Throughout the R\&D of this application, we have developed a useful set of toolbox of silicon micromachining and designing, and have used it for laterally spread applications such as tunable wavelength filter, fiber optic endoscope and fast optical scanner for laser image projection. Besides silicon-based MEMS, we also have developed a roll-to-roll printed version of large area MEMS technology for a flexible color pixel sheet that could be used for an electronic signage application. In this paper, we give an overview of optical MEMS R\&D and foresee diverging applications.

MOEMS and Miniaturized Systems X, edited by Harald Schenk, Wibool Piyawattanametha, Proc. of SPIE Vol. 7930, 793003 - @ 2011 SPIE · CCC code: 0277-786X/11/\$18 · doi: 10.1117/12.882725 


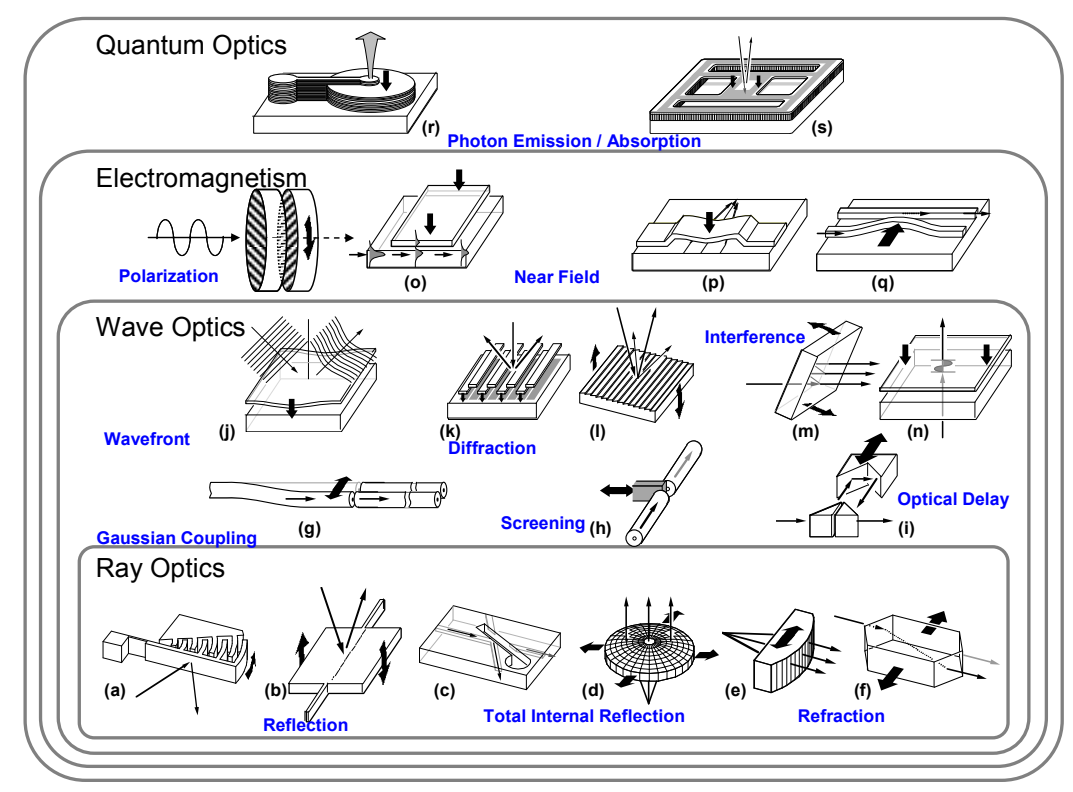

Figure 1 Light modulation by means of micromechanical motion.

\section{MEMS FOR FIBER OPTIC COMMUNICATION}

There was a boom of optical MEMS R\&D for emerging fiber telecom application in late 1990's and more than a hundred of start-up companies were created. They were mostly targeting for large scale optical crossconnect (OXC) application that is used in the heart of long haul fiber optic communication. Wavelength number superposed in the WDM system at that time increased from 4 to 16 or larger, and OXC of large input and output port was sought for as an attractive application of the optical MEMS technology. Planar lightwave circuit (PLC) technology at that time was a competitor for MEMS but MEMS was superior in accommodating very large port counts such as 128 or higher. Nonetheless, it was difficult to diffuse the MEMS technology into market mainly due to the economic crisis at the beginning of this century.

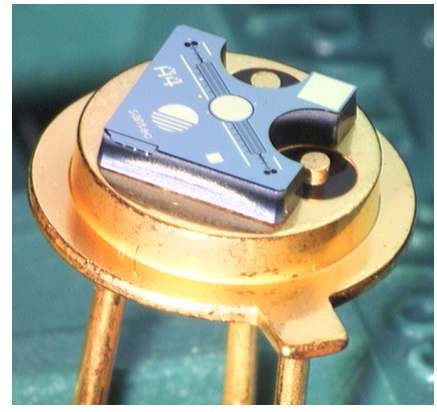

(a)

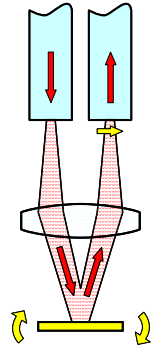

(b)

Figure 2 Electrostatic micro mirror for variable optical attenuator (VOA).

Despite such situation, MEMS technologies have found a place in small component industry of optics, where reduction of cost by integration was highly appreciated. Variable optical attenuator (VOA) was such application. Most conventional VOA used a motor-driven dimmer glass inserted between a pair of optical fibers, and hence it occupies large volume on a switching board particularly when they were designed to adapt to multiple wavelength application. To minimize the volume of the optical package and to lower the cost-per-bit, we have developed an SOI (silicon-on-insulator) bulk-micromachined micro mirror as shown in Figure 2 [4]. A mirror of $0.6 \mathrm{~mm}$ in 
diameter was made in one-piece with the electrostatic tilting mechanism and with a pair of torsion bars made of single crystalline silicon. We designed the MEMS structure and the process as simple as possible to transfer the design and processing technology from the R\&D lab to a MEMS foundry industry as soon as possible. Only two photolithography steps were used to perform deep-reactive ion etch (DRIE) the top and bottom surfaces of the wafer. Various reliability issues were cleared before commercialization, such as low voltage operation $(5 \sim 12 \mathrm{~V})$, high tolerance against external mechanical shock, temperature dependent drift, and electrostatic charge-up drift. Technical solutions for these problems were reported in detail in a reference [5].

VOAs are used in every place in the fiber telecom network. Figure 3 schematically illustrates the signal stream from the laser light source to the remote destination through an OXC. VOAs are needed to adjust the intensity of wavelengths before merging into one fiber, to equalize the intensity profile before and after $\mathrm{OXC}$, to reroute / block-out the malfunctioning section, and to adjust the intensity before received by a photodiode. Every optical component has its own rating optical power, and VOAs are used to manage the entire fiber network at its best performance to minimum the bit error rate.

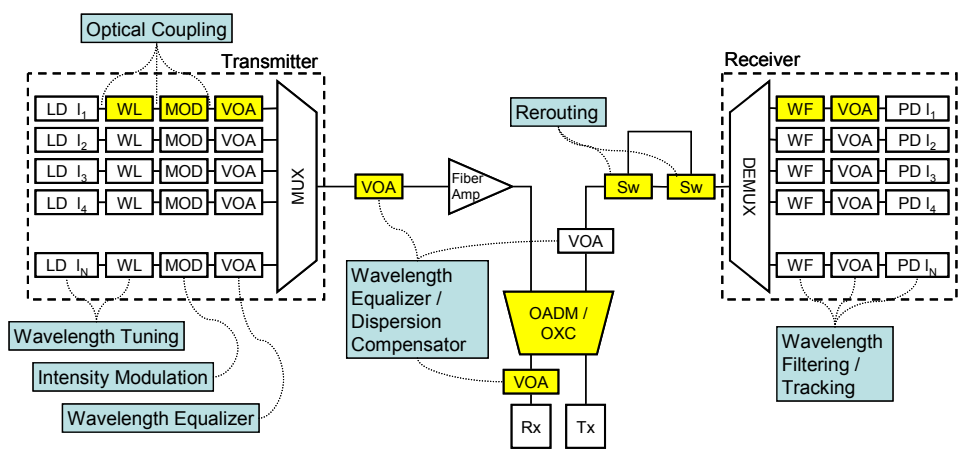

Figure 3 Schematic illustration of WDM fiber network, where various MEMS optical components are used.

Based on the SOI-micromachining technology developed through the VOA, we have used the skill to other optical MEMS devices. Figure 4 shows the scanning electron microscope (SEM) image of a wavelength tunable FabryPerot etalon made by the hybrid combination of a pair of glass cubes with multi-layer dielectric reflector and an electrostatic MEMS actuator platform [6]. One of the mirror cubes was mounted on an electrically movable stage and the Fabry-Perot gap of around 1 micron was controlled. A pair of input and output fibers was placed outside of the chip to construct a wavelength tunable filter. This was not a fully integrated MEMS mechanism but we chose the hybrid approach because superior optical performance was obtained on the dielectric coated glass mirror, which was processed separately from the MEMS micromachining. This sub-mount technique was used later to develop an MEMS optical scanner of high power handling [7].

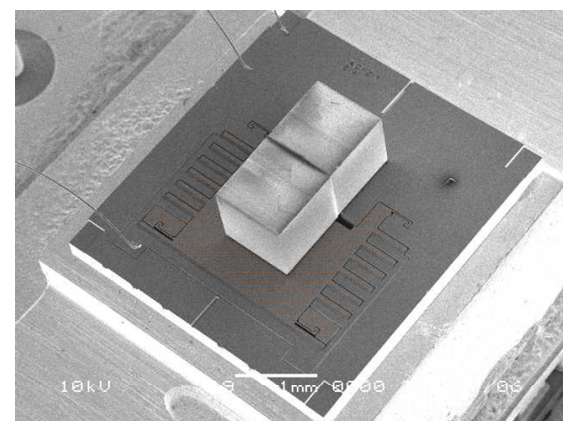

Figure 4 A pair of micro cube mirrors with multilayer dielectric coating. The gap between the cubes is controlled by the electrostatic actuator to tune the peak wavelength of the transmission light. 


\section{MEMS FOR OCT ENDOSCOPE}

The optical scanner in Figure 5 is an example of lateral spread application of optical MEMS, which is a beam steering device for a fiber optic endoscope [8]. A $1 \mathrm{~mm}$ x $1 \mathrm{~mm}$ square mirror of silicon was electrostatically actuated at the resonant frequency $(300 \mathrm{~Hz})$ by using the vertical comb-drive mechanism. This optical scanner was developed for an optical endoscope to steer the probe light in human body to obtain cross sectional image by the optical coherence tomography (OCT).

Figure 6(a) schematically illustrates the exploded view of the endoscope probe head. The endoscope developed here was supposed to be used in the blood vessel to visually inspect the cross section of the vessel walls near the heart. Therefore, electrical leakage or electromagnetic coupling with other medical instruments should be carefully avoided to minimize the risk of electrocution. For this reason, we have adopted the WDM approach to construct an optically driven endoscope. We used a $10 \mathrm{~mW}$ infrared light at 1.55 micron wavelength to supply power to the endoscope probe head, where the light energy is converted into electrical voltage by using a photovoltaic cell [9]. The powering light is chopped at the resonant frequency of the optical scanner such that the intensity modulated voltage signal can excite the scanner at the maximum scan angle. At the same time, another light at 1.3 microns was superposed into the identical fiber to probe the tissue. This probe light is spatially scanned over the tissue in the transverse direction. The depth information is obtained by the OCT optical interference measurement the other end of the fiber, where two-dimensional cross sectional image is reconstructed by using a PC. Figure 6(b) shows a photograph of an assembled package of the optical endoscope head. Optical components used in this package, such as a GRIN lens fiber, focusing lens, and dichroic mirror were originally developed for fiber telecom applications. From an OCT point of view, shorter wavelength around $900 \mathrm{~nm}$ is more suitable to see through the tissue with minimum absorption loss by water and hemoglobin; nonetheless, this was the first demonstration of optically driven MEMS endoscope as a byproduct of WDM application.

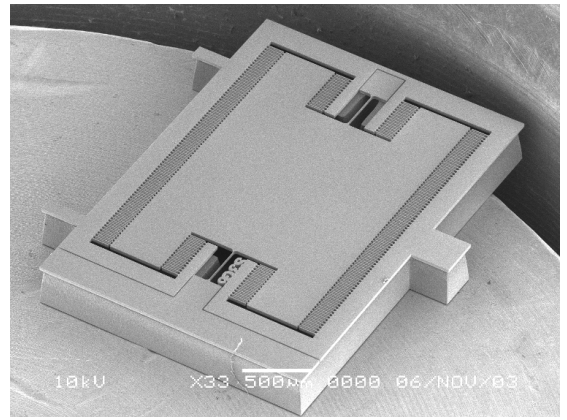

Figure 5 Electrostatic resonant scanner with electrostatic vertical-comb drive electrodes for fiber optic endoscope application.

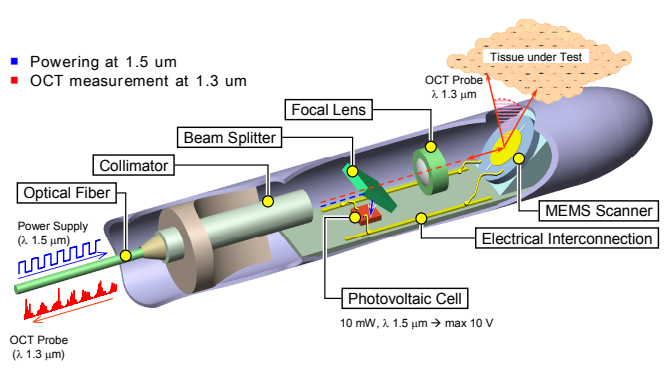

(a)

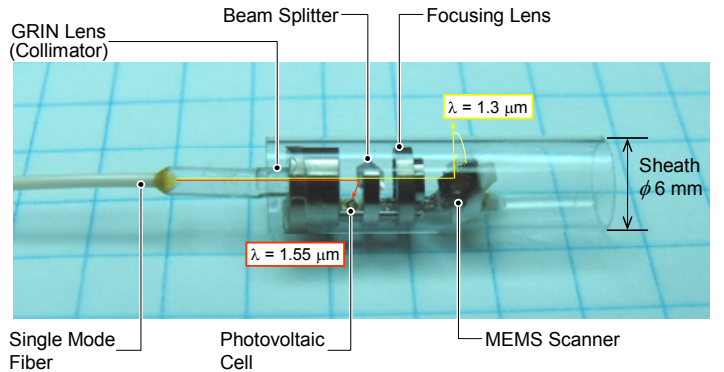

(b)

Figure 6 Schematic illustration of all-optical fiber endoscope and developed endoscope assembly.

Another optical MEMS component is included in this MEMS OCT system. Figure 7(a) shows the SEM image of high-speed optical scanner developed for the wavelength tuning of OCT light source. The round shape in the middle is a scanning mirror, which is accompanied with a pair of identical square actuator plates on the sides. 
Scanner's oscillation angle and operation frequency are usually constrained to a trade-off relationship and having both large and fast operation is usually difficult. To overcome this problem, we have developed an angle magnifying mechanism with silicon-based MEMS. The three-mass four-spring system in the figure is regarded as a two-degrees-of-freedom oscillation system, which has a mechanical effect to amplify the actuation displacement of the plate to deliver large angler displacement to the mirror [10]. A simple fabrication process was used to developed the offset vertical comb, as shown in Figure 7(b) and (c), by using the surface stiction force during the drying phase of the microfabrication process [11]. Thanks to the fast sweep frequency of wavelength, the image quality obtained by the OCT measurement has been improved dramatically as shown in Figure 8 . It has also an effect to improve the frame rate of the image, and we have recently succeeded in observing a cross-sectional image of a living animal in motion [12].

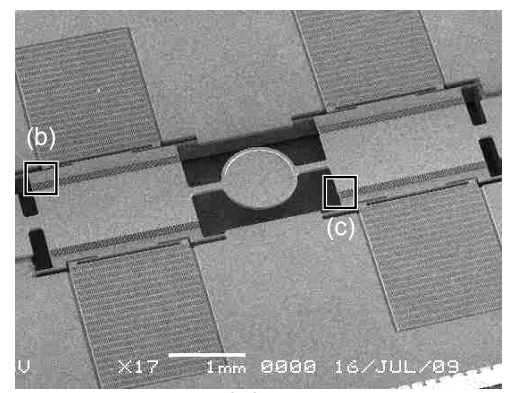

(a)

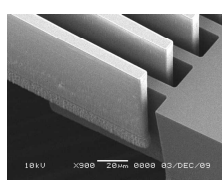

(b)

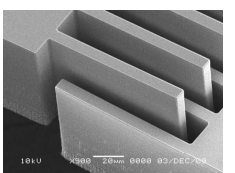

(c)

Figure 7 High frequency MEMS optical scanner with 2-DOF resonators.

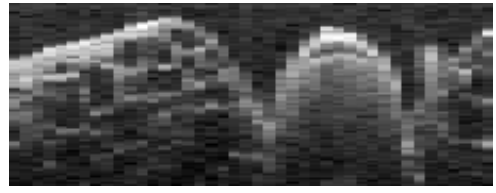

(a)

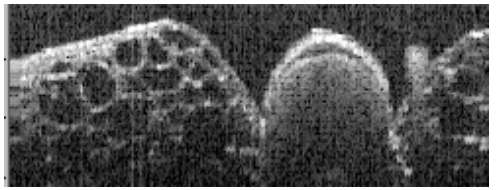

(b)

Figure 8 Improvement of OCT image quality.

\section{MEMS FOR IMAGE PROJECTION DISPLAY}

Consumer electronics have much larger market for optical MEMS; for instance, palmtop size image projection display is now driving the R\&D. Thanks to the collimation of a laser light, image screen can be placed in any distance from a projector, and hence the projector optics can be free from a focusing unit to make small package. MEMS optical scanners based on the electromagnetic operation has been already commercially released. Apart from it, we used PZT piezoelectric drive mechanism to construct yet smaller optical package, which is free from a permanent magnet unit usually occupying a considerable volume in electromagnetic MEMS scanners. Figure 9 shows a prototype module of such image projector with a PZT optical scanner [13]. SOI wafer of 30 to 50 micron thick active layer was used to deposit the PZT film by the ADRIP method [14]. The PZT layer is laminated with the top and the bottom metallic electrodes, and it gives either compressive or tensile stain depending upon the polarity of applied voltage. Figure 10 schematically illustrates the principle of the unimorph PZT actuation. 


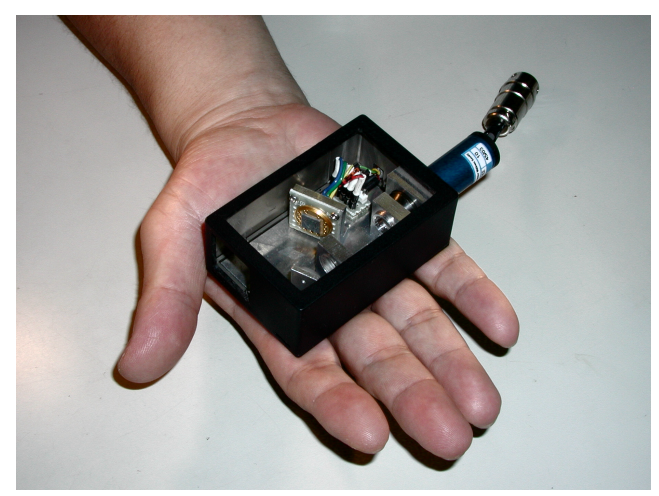

Figure 9 Palmtop image projector with 2D MEMS optical scanner.

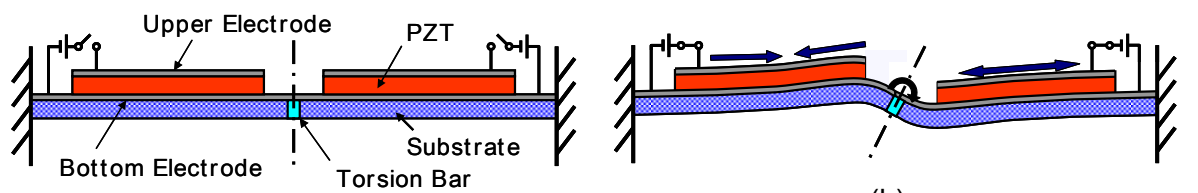

(a)

(b)

Figure 10 Actuation principle of PZT unimorph actuator beam.

Figure 11 shows the SEM image of a PZT scanner. The mirror in the middle is $1 \mathrm{~mm}$ in diameter, supported by a pair of torsion springs of silicon connected to the U-shape PZT unimorph actuators. The square inner gimbal is supported by the meandering electrodes to generate the out-of-plane motion in the orthogonal direction. The inner and outer axes had resonant frequency of around $1 \mathrm{kHz}$ and $340 \mathrm{~Hz}$, respectively, that corresponded to the fast horizontal scan and vertical scan. PZT operation of maximum $20 \mathrm{~V}$ delivered a peak-to-peak optical angle of 40 degrees. With this mechanism, we scanned three laser beams to project video graphic array (VGA) equivalent motion image as shown in Figure 12. The image size was about $40 \mathrm{~cm}$ in the horizontal direction, projected on the screen at a $1 \mathrm{~m}$ distance from the scanner.

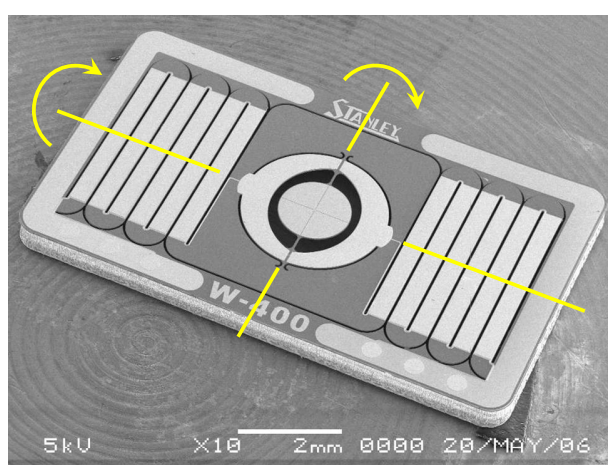

Figure 11 MEMS 2D optical scanner with PZT actuators. 

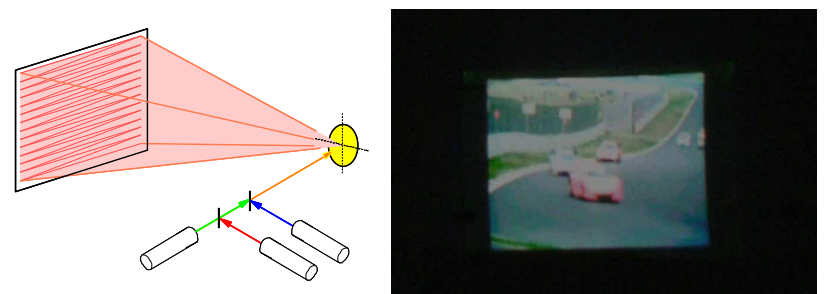

Figure 12 Demonstration of laser projection display with MEMS 2D optical scanner.

As a variation of MEMS projector, we have developed a three-dimension bitmap display [15]. Strong electrical field of YAG laser is known to strip electrons from molecule and to ignite a plasma light in air or in a liquid (laser breakdown mechanism), which was used as a bitmap light source for three-dimensional image display. Figure 13 shows the optical apparatus with three spatial modulators to position the focal spot in a liquid. The lens scanner placed after the YAG laser outlet is used to control the depth of the beam spot, while the pair of X and Y scanning mirror control the transverse location. Water was used in our experiment ignite the plasma at lower threshold power (4 mJ / $20 \mathrm{~ns}$ pulse at $1.064 \mathrm{um}$ ) that that of gaseous media. Figure 14 shows a snapshot of a 3D image demonstration in water container of $1 \mathrm{~cm} \mathrm{x} 1 \mathrm{~cm}$ and $5 \mathrm{~cm}$ in height. A series of plasma bitmap showed a 3D Lissajous image in water. As the plasma of water has a wide spectrum in the visible wavelength range, it could be seen in color through a color-changing TFT filter.

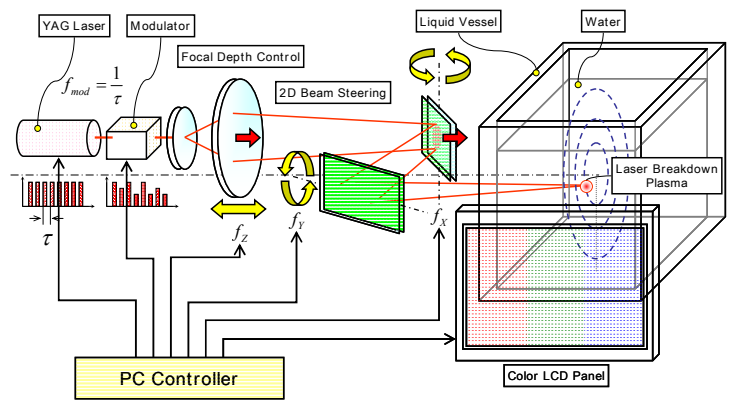

Figure 13 Optical apparatus for 3D bitmap image display based on laser breakdown effect.

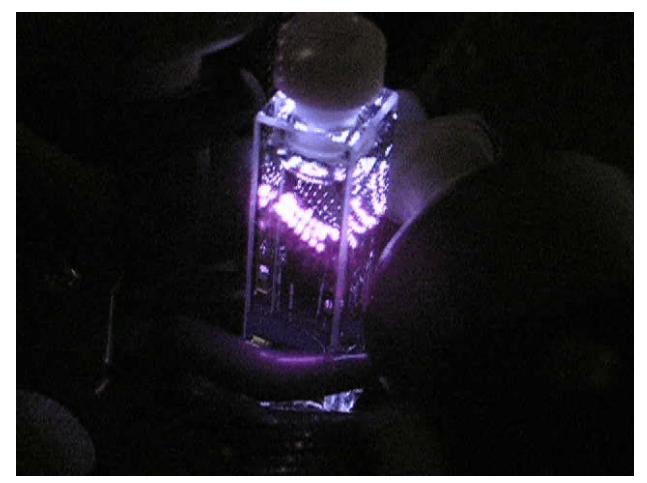

Figure 14 Demonstration of 3D bitmap image display based on laser breakdown in water.

The YAG laser used in the 3D display experiment had strong output power, and usual MEMS scanners with a metallic reflector on silicon were found to be easily burned out after a few seconds operation. Evaporated gold on a CMP-finished silicon surface has reflectively of around $95 \%$ at the infrared of $1.06 \mathrm{um}$, which means that $5 \%$ of the impinging power is converted into heat to thermally damage the mirror surface. For this reason, we have 
developed a MEMS optical scanner of high reflectivity by using the hybrid assembly technique [7]. As shown in Figure 15, we used a dielectric mirror deposited on a quartz substrate, and cut it into a $1.9 \mathrm{~mm} \times 2.9 \mathrm{~mm}$ piece by using the dicing saw. The backside of the mirror chip had a cross-shaped groove (also made by dicing) to mate with the silicon-based scanner platform without causing positioning mismatch. Due to the added mass of the mirror cube, the resonant frequency of the scanner platform was lowered to $570 \mathrm{~Hz}$ form the original value of 5.8 $\mathrm{kHz}$; the optical scan angle was increased, on the other hand, to more than 20 degrees at $50 \mathrm{Vac}$.

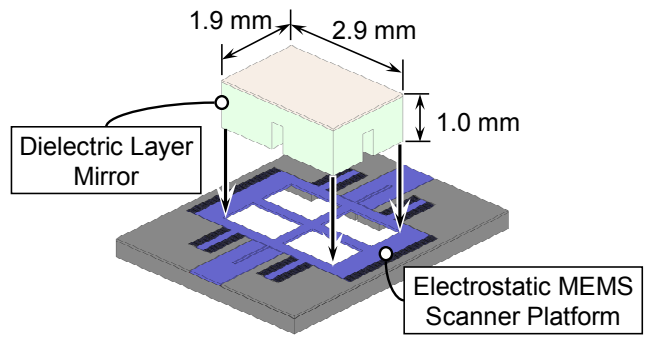

(a)

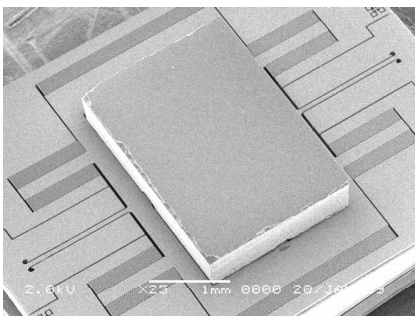

(b)

Figure 15 Optical apparatus for 3D bitmap image display based on laser breakdown effect.

\section{LARGE-AREA MEMS FOR FLEXIBLE DEVICES}

A flexible color-changing film was needed to let us observe the three-dimensional bitmap pixel in color. A flat color panel with TFT transistors was used for the first test (shown in Figure 13) but the viewing angle was limited to a small range. To increase the view angle for the 3D display, we have developed a flexible MEMS sheet that changed transmission color of light by means of electrostatically operated Fabry-Perot mechanism. Figure 16 shows a photograph of the developed film device that could be bent into a radius of curvature around $10 \mathrm{~cm}$ without losing the color-changing capability [16]. Detail operation mechanism is schematically shown in Figure 17. The device is made of two films; the bottom film is a 100 micron thick PEN (polyethylene naphthalate) film with a bottom metallic electrode (10 nm aluminum), an optical color interference layer (240 to $310 \mathrm{~nm}$ silicon oxide), and a 0.6 micron thick photoresist patterns as a spacer. The top film is a 16 micron thick PEN with another $10 \mathrm{~nm}$ aluminum film deposited on the bottom surface. The laminated films construct an electrostatic actuator to close the air gap by the attractive force of the applied voltage.

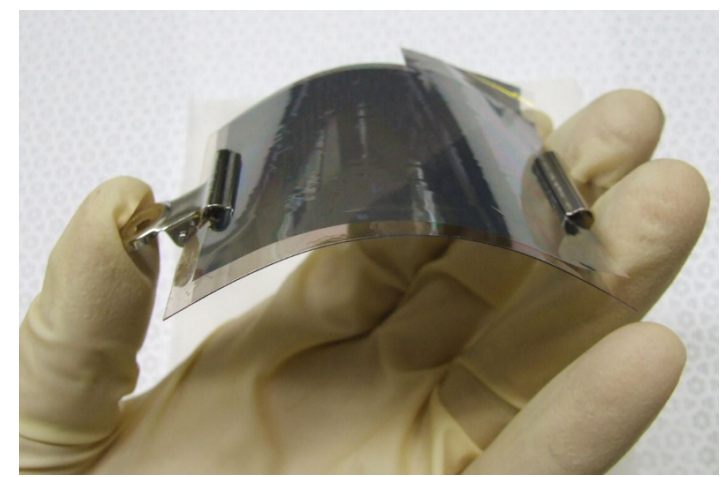

Figure 16 Large area flexible color sheet by plastic MEMS process. 


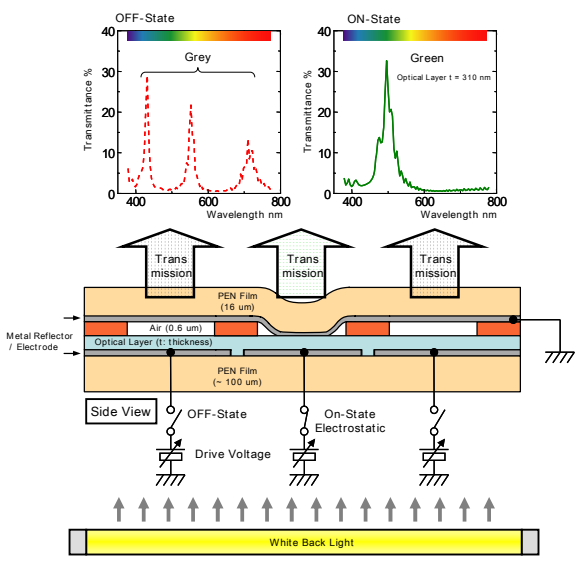

Figure 17 Electrostatically operated Fabry-Perot etalon.

The optical layer structures were designed to show in grey on a white backlight, when no voltage is applied. When voltage of around 40 to $100 \mathrm{~V}$ was applied, the upper PEN film was brought into contact with the bottom film to change the optical cavity. The color seen at this state depended on the thickness of the optical interference layer, and a vivid green was seen on a 310-nm-thick silicon oxide film. Figure 18 shows the color pixel in operation. Due the distributed deflection of the upper membrane, Newton rings were seen at the beginning of the electrostatic operation. After the electrostatic pull-in, the effective color area spread toward to the edges of the pixel. We have developed different pixel sizes ranging from 200 microns to 1000 microns, and found that the larger pixel had more effective area. Electrostatic latching operation was possible at a voltage lower than the pullin operation. Switching speed was found in several tens of ms range. Reliability issue was investigated and found electrostatic wear-out of the upper electrodes after several tens thousand contact operation. Studies are on-going on this device to develop a low-cost electronic signage.

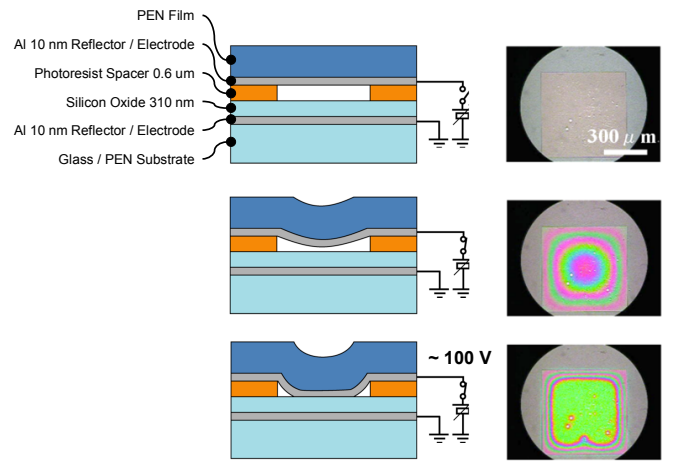

Figure 18 Demonstration of electrostatic Fabry-Perot color pixel.

Measurement results of color purity are shown in the CIE-1931 chart in Figure 19. Due to the poor reflectivity of the aluminum reflector/electrode used in the experiment, the color range that could be shown by this device was small in area compared with that of typical CRT fluorescent materials. By replacing the reflector material from aluminum to silver, we have recently demonstrated improved color quality [17]. 


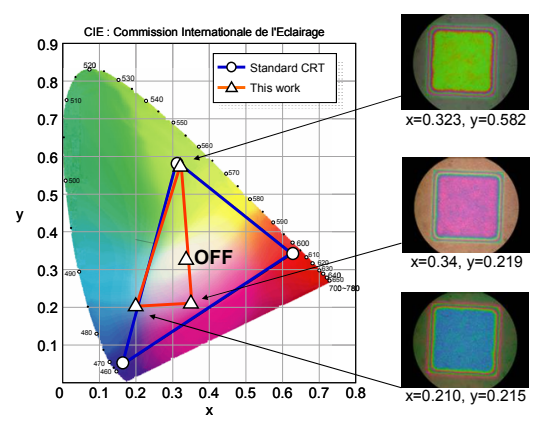

Figure 19 CIE-1931 color chart.

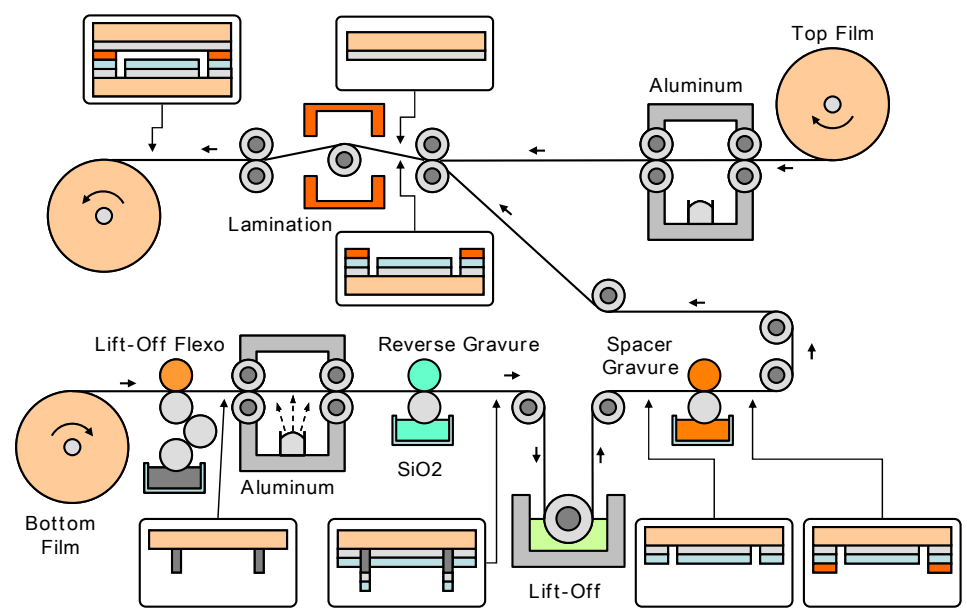

Figure 20 Roll-to-roll printing process to develop flexible color sheet based on the Fabry-Perot etalon.

So long as we use the semiconductor fabrication process based on the photolithography, the device size that could be developed is limited to the wafer size (4 inch in our case). To enlarge the device size and to lower the production cost, we have tested a printed version by using the roll-to-roll printing technology as shown in Figure 20. The bottom PEN film supplied from a reel is firstly processed by the lift-off printing module to put ink-mold patterns. Subsequently, an aluminum or silver metal film is deposited onto the surface, followed by the gravure printing of the optical interference layer. Patterning of the bottom electrodes are done in the same manner as liftoff, by selectively removing the ink-mold in the organic solvent. The air gap spacer is formed by using another gravure printing before laminating with the top film. Figure 21 shows the developed flexible color sheet example. The top and bottom films have an orthogonal set of line-and-space patterns of $1 \mathrm{~mm}$ electrodes such that the overlapped cross point can be electrically actuated. We have mixed black carbon powder in the spacer ink to block out the stray light from the pixel fringes. Electrostatic operation at voltage less than $10 \mathrm{~V}$ was possible by using a thinner plastic film ( $\sim 10$ microns) and by extending the pixel area to $2 \mathrm{~mm}$. Besides the electrical addressing of individual pixels, we also have tested manual addressing of pixel by physically pressing the upper film with a finger under medium bias voltage range $(\sim 10 \mathrm{~V})$. It was also found that the finger-pressed area was brought into the pull-in contact and the state was electrostatically latched for a few minutes. This indicated that the device could be used for a new application such as a hand-drawn chalk board [18]. 


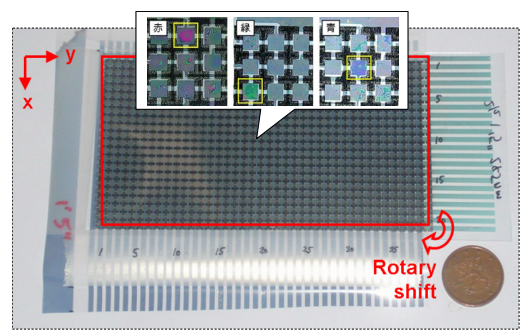

Figure 21 Example of roll-to-roll processed color pixel sheet.

\section{CONCLUSION}

In this paper, we have seen various applications of optical MEMS spatial light modulator (mainly mirror scanners) in fiber optic communication, medical endoscope and image display. As the progress of micromachining design and fabrication technologies, MEMS optical modulators are becoming commodity devices that we can even purchase in market, and hence the research activities on optical MEMS in academia have shifted toward more scientific side of MEMS to explore a new operation principle; for instance, optical MEMS devices based on subwavelength effect or plasmon effect are studied [19]. In parallel with such leading edge research, we also pursue system level R\&D of optical MEMS to create new service or entertainment gears, as illustrated in Figure 22 . For instance, combination of MEMS retinal scanner with a MEMS gyroscope would make a 4-pi display that a viewer can rotate the head to see image in every direction. It also can be used to optically suppress the image blur of camera. MEMS scanner can be used as a laser range finder to tell the distance to the screen such that the image can be corrected or controlled by the screen distance or attitude. Such range finder function has many other applications including the landing instrument for spacecraft, inter-vehicle communication, robot vision, 3D profile measurement optics, home security systems, gesture recognition optics, pier-to-pier free space optical link, and active homing device for visitors. Transistors have been turned into a commodity device but have wide range of applications from analog amplifiers to digital electronics. MEMS spatial light modulator would become like transistor, and its application is limited only by the imagination of users.
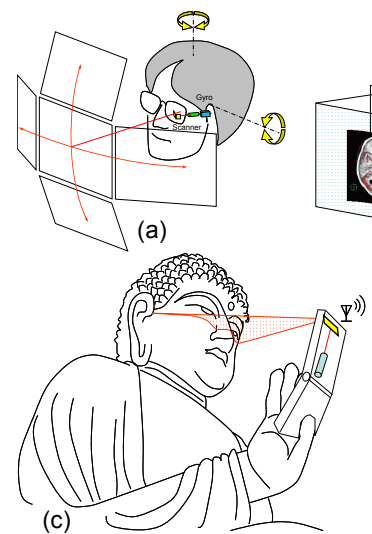

(c)

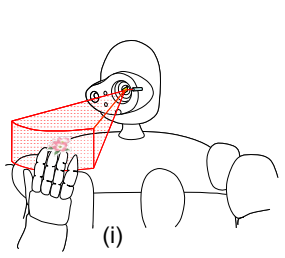

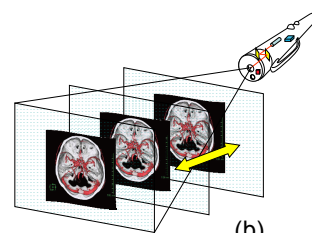

(b)

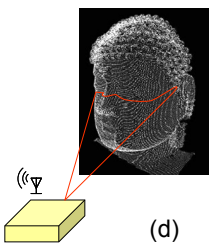

(d)

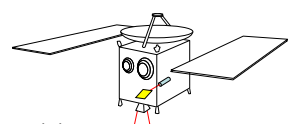

(e)

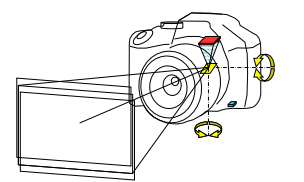

(g)
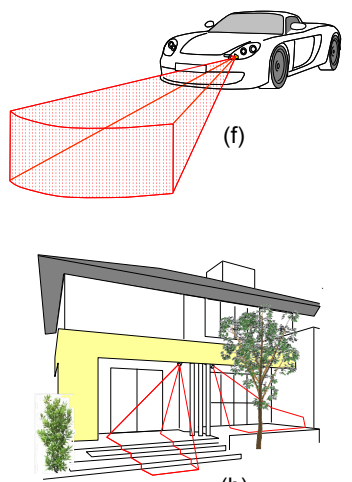

(h)
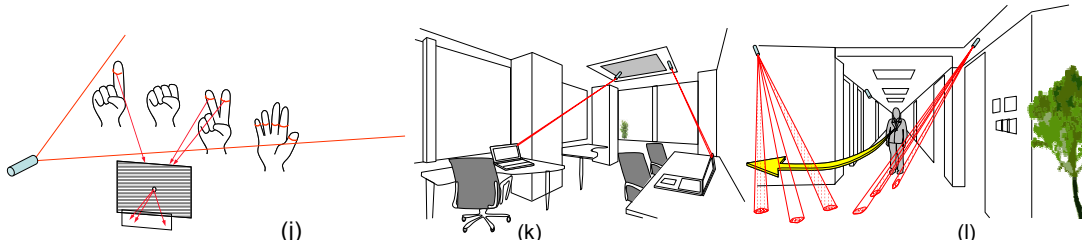

Figure 22 Applications of MEMS optical scanner. 


\section{REFERENCES}

[1] R. N. Thomas, G. J. Guldberg, H. C. Nathanson, P. R. Malmberg, "Mirror-matrix Tube - Novel Light Valve for Projection Displays," IEEE Trans. ED 29 (2), 765-775 (1975).

[2] D. R. Collins, J. B. Sampsell, L. J. Hornbeck, J. M. Florence, P. A. Penz, and M. T. Gately, "Deformable mirror device spatial light modulators and their applicability to optical neural networks," Applied optics 28 (22), 49004907, (1989).

[3] P. F. van Kassel, L. J. Hornbeck, R. E. Meier, and M. R. Douglass, "A MEMS-based Projection Display," Proc IEEE 86 (8), 1687-1704 (1998).

[4] K. Isamoto, A. Morosawa, M. Tei, H. Fujita and H. Toshiyoshi, "A 5-volt operated MEMS variable optical attenuator by SOI bulk micromachining," IEEE J. Selected Topics in Quantum Elec. (JSTQE) 10 (3), 570-578 (2004).

[5] K. Isamoto, C. Chong, and H. Toshiyoshi, "Reliability of MEMS VOA," a chapter contributed to O. Tabata, T. Tsuchiya, "Reliability of MEMS" (Wiley-VCH, Weinheim, 2007).

[6] T. Yamanoi, T. Endo, and H. Toshiyoshi, "A hybrid-assembled MEMS Fabry-Perot wavelength tunable filter," Sensors and Actuators A 145-146, 116-122 (2008).

[7] Y. Ohira, A. Chekhovskiy, T. Yamanoi, T. Endo, H. Fujita, and H. Toshiyoshi, "Hybrid MEMS optical scanner for volumetric 3-D displays,” Journal of SID 17 (5), 419-422 (2009).

[8] M. Nakada, C. Chong, A. Morosawa, K. Isamoto, T. Suzuki, H. Fujita, and H. Toshiyoshi, "Optical coherence tomography by all-optical MEMS fiber endoscope," IEICE Electronics Express 7 (6) 428-433 (2010).

[9] C. Chong, K. Isamoto, and H. Toshiyoshi, "Optically Modulated MEMS Scanning Endoscope," Photon. Tech. Lett. 18 (1), 133-135 (2006).

[10] M. Yoda, K. Isamoto, C. Chong, H. Ito, A. Murata, and H. Toshiyoshi, "Design and Fabrication of A MEMS 1-D Optical Scanner using Self-Assembled Vertical Combs and Scan-Angle Magnifying Mechanism," IEEE/LEOS Int. Conf. on Optical MEMS and Their Applications, August 1-4, 2005, Oulu, Finland.

[11] K. Isamoto, T. Makino, A. Morosawa, C. Chong, H. Fujita, and H. Toshiyoshi, "Self-Assembly Technique for MEMS Vertical Comb Electrostatic Actuators," IEICE Electronics Express 2 (9), 311-315, (2005).

[12] K. Isamoto, K. Totsuka, T. Sakai, T. Suzuki. A. Morosawa, C. Chong, and H. Toshiyoshi, "High speed MEMS scanner based swept source laser for SS-OCT," IEEJ Sensor Symposium, Oct. 14-15, Matsue, Shimane, Japan (in Japanese).

[13] M. Tani, M. Akamatsu, Y. Yasuda, and H. Fujita, and H. Toshiyoshi, "Two-Axis Piezoelectric Tilting Micromirror with a Newly Developed PZT-meandering Actuator," in Proc. 20th IEEE Int. Conf. on Micro Electro Mechanical Systems (MEMS 2007), Jan. 21-25, 2007, Kobe Portopia Hall and Kobe Portopia Hotel, Japan.

[14] M. Akamatsu et al., "Fast Deposition of Lead-Zirconate-Titanate Thick Films by Arc Discharged Reactive-Ion Plating Method," Jpn. J. Appl. Phys. 41, 6976-6979 (2002).

[15] A. Chekhovskiy and H. Toshiyoshi, "A use of laser burst for volumetric imaging inside of transparent liquid," Jpn. J. Appl. Phy. 47 (8), 6790-6793 (2008).

[16] Y. Taii, A. Higo, H. Fujita and H. Toshiyoshi, “A transparent sheet display by plastic MEMS,” J. SID 14 (8), $735-$ 741 (2006).

[17] C.-Y Lo, O.-H. Huttunen, J. Hiitola-Keinanen, J. Petaja H. Fujita, and H. Toshiyoshi, "MEMS-Controlled PaperLike Transmissive Flexible Display,” IEEE J. Microelectromech. Syst. 19 (2), 410-418 (2010).

[18] R. Shigematsu, A. Higo, H. Toshiyoshi, and H. Fujita, "An electrostatically latched and magnetically erased MEMS re-writable bitmap image display," IEICE Electronics Express 3 (5), 87-91 (2006).

[19] T. Lee, A. Higo, H. Fujita, Y. Nakano, and H. Toshiyoshi, "A study on color-tunable MEMS device based on plasmon photonics," in Proc. IEEE Int. Conf. on Optical MEMS and Nanophotonics, Sapporo Convention Center, Sapporo, Japan, Aug. 9-12, 2010, pp.107-108. 